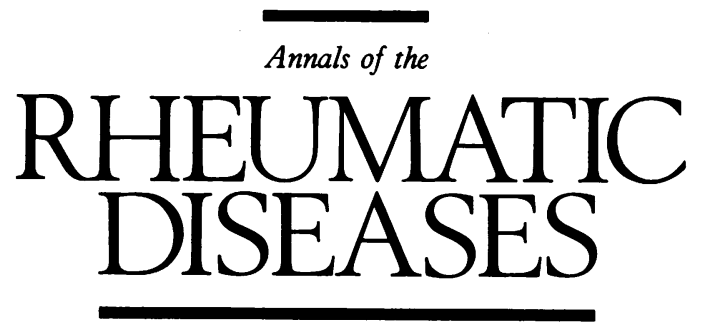

Leader

\title{
The ANCA test: its clinical relevance
}

In theory, the diagnosis of vasculitis depends on the presence of certain clinical features and the demonstration of vascular disease by either biopsy or angiography. ${ }^{1}$ In practice, there have been many difficulties in formulating a satisfactory classification of the vasculitides. In Wegener's granulomatosis, for example, where there are prominent pulmonary changes, infections, other granulomatous conditions, and neoplastic diseases may produce a similar clinical picture, and biopsy specimens are often nondiagnostic. ${ }^{2}$ The demonstration that antineutrophil cytoplasmic antibodies (ANCAs) are present in most cases of Wegener's granulomatosis has provided a useful diagnostic tool and may shed light on the pathogenesis of vasculitis. ${ }^{3}$

Ten years ago, ANCAs were found in a small number of patients with glomerulonephritis. ${ }^{45}$ In 1985 van der Woude et al demonstrated the high sensitivity of ANCA in the diagnosis of Wegener's granulomatosis and a correlation with disease activity. ${ }^{3}$ These findings were corroborated by other groups. ${ }^{67}$ Indirect immunofluorescence is the most widely accepted method for detecting ANCAs, and a standardised methodology has been recently adopted. ${ }^{8}$ So far, two different patterns have been identified: classical ANCA, which produces a coarsely granular, centrally accentuated immunofluorescence and perinuclear ANCA, which gives a perinuclear staining pattern. The significance of other patterns, such as a diffuse cytoplasmic immunofluorescence, is unclear at present.

Antineutrophil cytoplasmic antibody reacts against cytoplasmic antigens of neutrophils and monocytes of human origin but not those from other species. Leucocyte alkaline phosphatase was initially thought to be the target antigen. ${ }^{9}$ Recent investigations, however, have shown that classical ANCA is directed against a soluble, single chain $29 \mathrm{kD}$ protein contained in the primary or azurophilic granules of the neutrophils and in the lysosomal granules of monocytes. ${ }^{10} 11$ This is most likely to be proteinase 3, a serine protease. ${ }^{12}$ Perinuclear ANCA reacts mainly with myeloperoxidase but also with elastase, both of which are contained in the primary granules of the neutrophil. ${ }^{1314}$ The different staining pattern is due to an artefactual staining of alcohol fixed neutrophils, but in formalin fixed neutrophils the same antibodies produce a cytoplasmic staining similar to classical ANCA. ${ }^{13}$ The previous observations suggest that ANCAs represent a novel class of autoantibodies directed against proteinase 3, myeloperoxidase, elastase, and, probably, other enzymes present in the lysosomal granules of neutrophils and monocytes.

Enzyme linked immunosorbent assays (ELISAs) using purified primary granules of neutrophils or myeloperoxidase as antigens have been developed. ${ }^{13} 15$ They permit a more objective quantitative determination of classical and perinuclear ANCA respectively. The diagnostic senstivity of the
ELISA seems to be as high as that of indirect immunofluorescence. An ELISA may also detect ANCA in serum samples from patients with Wegener's granulomatosis and negative indirect immunofluorescence titres, and is useful in cases where there are difficulties in reading immunofluorescence patterns because of simultaneous antinuclear antibodies, which may obscure the cytoplasmic staining. The use of purified antigens also results in greater specificity than previous ELISAs and radioimmunoassays which used crude antigen preparations. ${ }^{6}$ As the indirect immunofluorescence recognises the different types of ANCA it is quite likely that this will remain the preferred screening test.

What is the clinical significance of ANCA? There is no doubt that determination of ANCAs is a valuable test in the diagnosis of Wegener's granulomatosis, as they are present in 50 to $96 \%$ of all patients with this diagnosis. ${ }^{6}$ Its sensitivity is 78 to $100 \%$ in active and disseminated Wegener's granulomatosis, 60 to $70 \%$ in cases with disease limited to the respiratory tract, and roughly $30 \%$ in patients with Wegener's granulomatosis in remission. The specificity of the test in the diagnosis of Wegener's granulomatosis has been consistently high (90 to $100 \%){ }^{7} 16$ The presence of ANCA does not absolve the doctor from actively pursuing a histological diagnosis, however. Most proved cases of Wegener's granulomatosis show a classical ANCA pattern. Titres of ANCA may fluctuate with the clinical activity of the disease and decrease or disappear with treatment. ${ }^{7} 16$ Classical ANCA has also been found in microscopic polyarteritis nodosa ${ }^{6}$ and in Kawasaki disease, ${ }^{17}$ though in the latter the immunofluorescence pattern is not quite typical. Therefore ANCA determination is not only a good marker for the presence and activity of the disease, but may also be helpful in distinguishing between exacerbations of the disease and infection, a common complication in Wegener's granulomatosis.

The interpretation of perinuclear ANCA is somewhat less clear. It has been found associated mainly with necrotising and crescentic glomerulonephritis. ${ }^{1318}$ As up to two thirds of patients with crescentic glomerulonephritis have extrarenal vasculitic disease, and some of them have clinical disease also characteristic of Wegener's granulomatosis, the same authors have proposed that all patients with ANCA fall within a continuum of pathological features ranging from limited renal disease to varying degrees of systemic involvement.

Antineutrophil cytoplasmic antibody has been occasionally found in Churg-Strauss disease, Takayasu's disease, systemic lupus erythematosus, relapsing polychondritis, and Behçet's disease. ${ }^{19}$ Most of these studies have detected ANCA of the IgG class. Lockwood, however, identified a small group of patients with exclusively IgM ANCA, who developed pulmonary haemorrhage and severe glomerulonephritis. ${ }^{20}$ 
Recently, IgA ANCA has been reported in patients with Henoch-Schönlein purpura and IgA nephropathy. ${ }^{21}$

The question remains whether ANCA is simply a disease marker or has a pathogenic role. It has been proposed that immune complexes play a part in the pathogenesis of Wegener's granulomatosis. Circulating immune complexes have been detected in some patients, but their role is unclear as serum complement concentrations are not depressed and immunofluorescence studies have indicated deposits of immunoglobulins and complement in only few cases of Wegener's granulomatosis. ${ }^{22}$ Recent studies support the hypothesis that ANCAs are more than a useful serum abnormality. By electron microscopy, intravascular lysis of neutrophils has been found as an early event in the inflammatory process. ${ }^{23} \mathrm{~A}$ chemotactic abnormality has also been reported, ${ }^{24}$ as well as evidence of neutrophil activation in vitro ${ }^{25}$ and in vivo. ${ }^{26} \mathrm{IgG}$ from patients with antibody to myeloperoxidase inhibits the enzymatic activity of myeloperoxidase in a chemiluminescence system, implying a disturbance in neutrophil function. ${ }^{27}$ Furthermore, it has been shown that ANCA might enter live neutrophils, ${ }^{3}$ and ANCA antigen may be expressed in the cellular membrane or be released from neutrophils stimulated with cytochalasin $B$ in vitro. ${ }^{11}$ For many years it has been thought that Wegener's granulomatosis is an immunological reaction triggered by a specific antigen. The lung, an organ exposed to the outer environment and commonly affected in Wegener's granulomatosis, has unique anatomical and physiological features with a large marginated pool of leucocytes. ${ }^{28}$ Conceivably, a triggering event such as infection might lead to activation of leucocytes, and the presence of ANCA might tilt the balance toward frank neutrophil activation, release of lysosomal enzymes, production of oxygen metabolites, and tissue injury. Reports of the beneficial effects of sulphamethoxazole-trimethoprim on the course of the disease lend additional support to this theory. ${ }^{29}$ Theoretically and practically it is interesting that high dose intravenous immunoglobulin contains inhibitory antibodies reactive with ANCA. ${ }^{30}$ Studies of ANCA in the interaction of neutrophils with endothelial vascular cells may help to clarify its importance in the pathogenesis of vasculitis.

Using a monoclonal antibody against the neutrophil cytoplasmic antigen, Abbott et al detected antigenic determinants on glomerular endothelial and epithelial cells, suggesting that such cross reactivity might initiate the development of vasculitis in patients with ANCA. ${ }^{31}$

Cellular immune mechanisms should not be ruled out in the pathogenesis of the disease. The presence of granulomas is characteristic of cellular immune disturbances. Immunochemical studies of biopsy specimens have shown that monocytes and $T$ lymphocytes are the dominant cell types in the cellular infiltrates. ${ }^{32}$ Recent studies emphasise the complex ways in which the $T$ lymphocyte may affect neutrophil function. ${ }^{33}$

Although too much importance may have been given to the discovery of ANCA and other tests providing evidence of active blood vessel injury, these are, nonetheless, welcome advances in the understanding of the pathogenesis of vasculitis. ${ }^{34}$

Dr Ramírez is recipient of a scholarship from the British Council.

Lupus Arthritis Research Unit,

G RAMÍREZ

The Rayne Institute,

St Thomas's Hospital,

HAMASHTA

London SE1 $7 E H$ GR V HUGHES

Correspondence to: Dr Hughes.
1 Cupps T R, Fauci A S. The vasculitides. Philadelphia: Saunders, 1981: 1-5. D'Cruz D P, Baguley E, Asherson R A, Hughes G R V. Ear, nose and throat symptoms in sub-acute Wegener's granulomatosis. $\mathrm{Br}$ Med $\mathcal{F} 1989 ; 299$ : 419-22.

3 van der Woude F J, Rasmussen N, Lobatto $S$, et al. Autoantibodies against neutrophils and monocytes: tool for diagnosis and marker of disease activity in Wegener's granulomatosis. Lancet 1985; i: 425-9.

4 Davies D J, Moran J E, Niall J F, Ryan G B. Segmental necrotising glomerulonephritis with antineutrophil antibody: Possible arbovirus aetiology? Br Med f 1982; 285: 606 .

5 Hall J B, Wadham B M, Wood C J, Ashton V, Adam W R. Vasculitis and glomerulonephritis: a subgroup with an antineutrophil cytoplasmic antibody. Aust NZ $\mathcal{F}$ Med 1984; 14: 277-8.

6 Savage C O, Winearls C G, Jones S, Marshall P D, Lockwood C M. Prospective study of radioimmunoassay for antibodies against neutrophi cytoplasm in diagnosis of systemic vasculitis. Lancet 1987; i: 1389-93.

7 Nolle B, Specks U, Ludemann J, Rohrbach M S, DeRemee R A, Gross W L. Anticytoplasmic autoantibodies: their immunodiagnostic value in Wegener's granulomatosis. Ann Intern Med 1989; 111: 28-40.

8 Wiik A. Delineation of a standard procedure for indirect immunofluorescence detection of ANCA. Acta Pathol Microbiol Immunol Scand 1989; 97 (suppl 6): 12-13.

9 Lockwood C M, Bakes D, Jones S, Whitaker K B, Moss D W, Savage C O. Association of alkaline phosphatase with an autoantigen recognized by circulating anti-neutrophil antibodies in systemic vasculitis. Lancet 1987; i: 716-20.

10 Borregaard N, Rasmussen N. Constituents of the human neutrophil cytoplasm: methods for examination and methodological problems with reference to the binding site of the anti-neutrophil cytoplasmic antibodies. Acta Pathol Microbiol Immunol Scand 1989; 97 (suppl 6): 10-11.

11 Calafat J, Goldschmeding R, Ringeling P L, Janssen H, van der Schoot C E. In situ localization of double-labeling immunoelectron microscopy of antineutrophil cytoplasmic autoantibodies in neutrophils and monocytes. Blood 1990; 75: 242-50.

12 Goldschmeding $\mathrm{R}$, van der Schoot C E, ten Bokkel Huinink D, et al. Wegener's granulomatosis autoantibodies identify a novel diisopropyfluorophosphate-binding protein in the lysosomes of normal human neutrophils. $\mathscr{f}$ Clin Invest 1989; 84: 1577-87.

13 Falk R J, Jennette J C. Anti-neutrophil cytoplasmic autoantibodies with specificity for myeloperoxidase in patients with systemic vasculitis and idiopathic necrotizing and crescentic glomerulonephritis. $N$ Engl $\mathcal{f} \mathrm{Med}$ 1988; 318: 1651-7.

14 Goldschmeding R, Cohen Tervaert J W, van der Schoot C E, van der Veen C, Kallenberg C G M, con dem Borne A E G Kr. ANCA, antimyeloperoxidase and antielastase: three members of a novel class of autoantibodies against myeloid lysosomal enzymes. Acta Pathol Microbiol Immunol Scand 1989; 97 (suppl 6): 48.

15 Rasmussen N, Sjolin C, Isaksson B, Bygren P, Wieslander J. An ELISA for the detection of anti-neutrophil cytoplasm antibodies (ANCA). $\mathcal{f}$ Immunol Methods 1990; 127: 139-45.

16 Specks U, Wheatley C L, McDonald T J, Rohrbach M S, DeRemee R A. Anticytoplasmic autoantibodies in the diagnosis and follow-up of Wegener's granulomatosis. Mayo Clin Proc 1989; 64: 28-36.

17 Savage C O S, Tizard J, Jayne D, Lockwood C M, Dillon M J. Antineutrophil cytoplasm antibodies in Kawasaki disease. Arch Dis Child 1989; 64: 360-3.

18 Jennette J C, Wilkman A S, Falk R J. Anti-neutrophil cytoplasmic autoantibody-associated glomerulonephritis and vasculitis. Am f Pathol 1989; 135: 921-30.

19 Harrison D J, Simpson R, Kharbanda R, Abernethy V E, Nimmo G. Antibodies to neutrophil cytoplasmic antigens in Wegener's granulomatosis Antibodies to neutrophil cytoplasmic antigens in

20 Lockwood C M. Autoimmune responses in systemic vasculitis. Am f Nephrol 1989; 9 (suppl 1): $30-2$.

21 van den Wall Bake A W L, Daha M R, van der Woude F J, et al. IgA class anti-neutrophil cytoplasmic antibodies (IgA-ANCA) in primary IgA nephropathy. Acta Pathol Microbiol Immunol Scand 1989; 97 (suppl 6): $25-6$

22 Howell S B, Epstein W V. Circulating immunoglobulin complexes in Wegener's granulomatosis. Am f Med 1976; 60: 259-68.

23 Donald K J, Edwards R L, McEvoy J D S. An ultrastructural study of the pathogenesis of tissue injury in limited Wegener's granulomatosis. Pathology 1976; 8: 161-9.

24 Niinaka T, Okochi T, Watanabe Y, Takahashi Y, Yamamura Y. Chemotactic defect in Wegener's granulomatosis. F Med 1977; 8: 161-75.

25 Falk R J, Terrell R, Huneycutt-Calder L, Jennette J C. Anti-neutrophil cytoplasmic autoantibodies (ANCA) stimulate neutrophil activation in vitro cytoplasmic autoantibodies (ANCA) stim
[Abstract]. Kidney Int 1989; 35: 346.

26 Wathen C G, Bell D, Harrison D J, Dawes J, Douglas A C. Neutrophil activation in Wegener's granuloma [Abstract]. Am Rev Respir Dis 1988; 137 (suppl): 348 .

27 Lee S S, Adu D, Thompson R A. Anti-myeloperoxidase antibodies in systemic vasculitis. Clin Exp Immunol 1990; 79: 41-6.

28 Hogg J C. Neutrophil kinetics and lung injury. Physiol Rev 1987; 67: $1249-90$.

29 DeRemee R A, McDonald T J, Weiland L H. Wegener's granulomatosis: observations on treatment with antimicrobial agents. Mayo Clin Proc 1985; 60: $27-32$

30 Jayne D R W, Lockwood C M. Pathogenesis of acute Kawasaki disease. Lancet 1990; i: 410-1.

31 Abbott F, Jones S, Lockwood C M, Rees A J. Autoantibodies to glomerular antigens in patients with Wegener's granulomatosis. Nephrology, Dialysis and Transplantation 1989; 4: 1-8.

32 Gephardt G, Ahmad M, Tubbs R R. Pulmonary vasculitis (Wegener's granulomatosis) immunohistochemical study of T and B cell markers. Am $\mathcal{J}$ Med 1983; 74: 700-4.

33 Campbell P A. The neutrophil, a professional killer of bacteria, may be controlled by T cells. Clin Exp Immunol 1990; 79: 141-3.

34 Smiley J D, Moore S E. Immune-complex vasculitis: role of complement and IgG-Fc receptor functions. Am $\mathcal{F}$ Med Sci 1989; 298: 267-76. 